\title{
Klinische Bedeutung von Bakterien beim chronischen Ulcus cruris
}

\author{
Clinical Relevance of Bacteria in Chronic Leg Ulcers
}

Autoren

Institut
W. Sondermann, J. Dissemond

Klinik und Poliklinik für Dermatologie, Venerologie und Allergologie, Universitätsklinikum Essen
Bibliografie

DOI http://dx.doi.org/

10.1055/s-0034-1377960

Akt Dermatol 2014; 40: 523-527

(c) Georg Thieme Verlag KG

Stuttgart · New York

ISSN 0340-2541

Korrespondenzadresse

Prof. Dr. med.

Joachim Dissemond

Universitätsklinikum Essen

Klinik und Poliklinik für

Dermatologie, Venerologie

und Allergologie

Hufelandstraße 55

45122 Essen

joachim.dissemond@uk-essen.de

\section{Zusammenfassung \\ $\nabla$}

Bakterien können bei Patienten mit chronischem Ulcus cruris sowohl in der Genese als auch als Komplikation von erheblicher Bedeutung sein. Am häufigsten wird bei mindestens $50 \%$ der Patienten Staphylococcus aureus nachgewiesen; am zweithäufigsten wird mit steigender Tendenz Pseudomonas aeruginosa gefunden. Eine zunehmende Bedeutung gewinnen Problemkeime wie Methicillin-resistente Stapylococcus aureus (MRSA) oder multiresistente gramnegative Erre-

\section{Einleitung}

In aktuellen Leitlinien wird empfohlen Wunden als „chronisch“ zu bezeichnen, wenn diese seit mindestens acht Wochen bestehen [1]. Grundsätzlich können chronische Wunden an jeglichen Lokalisationen des Körpers auftreten. Neben Dekubitus, diabetischem Fußsyndrom oder Wunden bei peripherer arterieller Verschlusskrankheit (pAVK) sind es insbesondere die Patienten mit chronischem Ulcus cruris, die in dermatologischen Institutionen behandelt werden.

Als Ulcus cruris werden Wunden bezeichnet, die am Unterschenkel lokalisiert sind. Somit beschreibt der Begriff Ulcus cruris lediglich ein Symptom und keine Diagnose. Erst durch einen weiteren Zusatz, der Auskunft über die Ätiologie gibt, wie beispielsweise Ulcus cruris venosum, wird das Krankheitsbild korrekt beschrieben. Zahlreiche weitere Faktoren, wie beispielsweise Vaskulitiden, Lymphödeme oder Medikamente, können ebenfalls ein chronisches Ulcus cruris verursachen oder dessen Abheilung zumindest wesentlich behindern. In Deutschland gibt es entsprechend aktueller Schätzungen zirka eine Million Patienten mit chronischem Ulcus cruris $[2,3]$. ger (MRGN). Für den Erregernachweis reichen meist bakteriologische Abstriche der Wundoberfläche beispielsweise entsprechend der Technik des „Essener Kreisels“.

In der überwiegenden Zahl der Patienten mit chronischem Ulcus cruris liegt eine unkritische bakterielle Kolonisation vor, sodass keine systemische Antibiotikatherapie notwendig ist. Für Patienten mit erhöhtem Infektionsrisiko sowie für Wunden mit lokalen Infektionszeichen sind Antiseptika mit Polihexanid oder Octenidin meist erste Wahl.

\section{Bakterien als Ursache eines chronischen Ulcus cruris}

Prädisponierende Faktoren für Pyodermien können beispielsweise heißes oder feuchtes Klima, mangelnde Hygiene, Mangelernährung, Adipositas, Diabetes mellitus, Immunsuppression oder Störung der Hautbarriere durch Ekzeme oder Mykosen darstellen.

Bei den Ekthymata handelt es sich um ulzerierende Pyodermien, die meist durch Superinfektionen vorbestehender Verletzungen mit Streptokokken oder Staphylokokken entstehen. Wenn Bakterien die Hautbarriere durchdringen, kommt es zu Pusteln auf erythematösem Grund ( $\bullet$ Abb. 1). Es bildet sich zentral eine Nekrose aus, die im weiteren Verlauf zu den typischen scharf begrenzten Ulzerationen mit Durchmessern von $2-5 \mathrm{~cm}$ führt ( $\bullet$ Abb.2) [2].

Die chronisch vegetierende Pyodermie beschreibt eine primär an Hautanhangsgebilde gebundene bakterielle Infektion der Haut, die sekundär zu Exulzerationen neigt. Die verursachenden Erreger sind meist Streptokokken oder Staphylokokken. Insbesondere die galenisch falsche Lokaltherapie, beispielsweise mit Panthenol-haltigen „Wundsalben“, begünstigt die Chronifizierung des Krankheitsbildes. Klinisch gestaltet sich aufgrund des aufgeworfenen Randwalls mit zentra- 


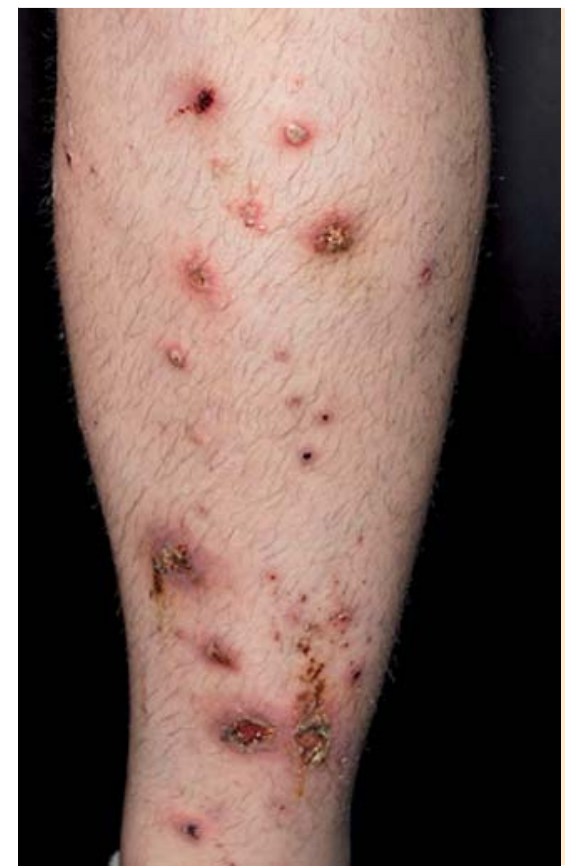

Abb. 1 Initialbefund einer ulzerierenden Pyodermie.

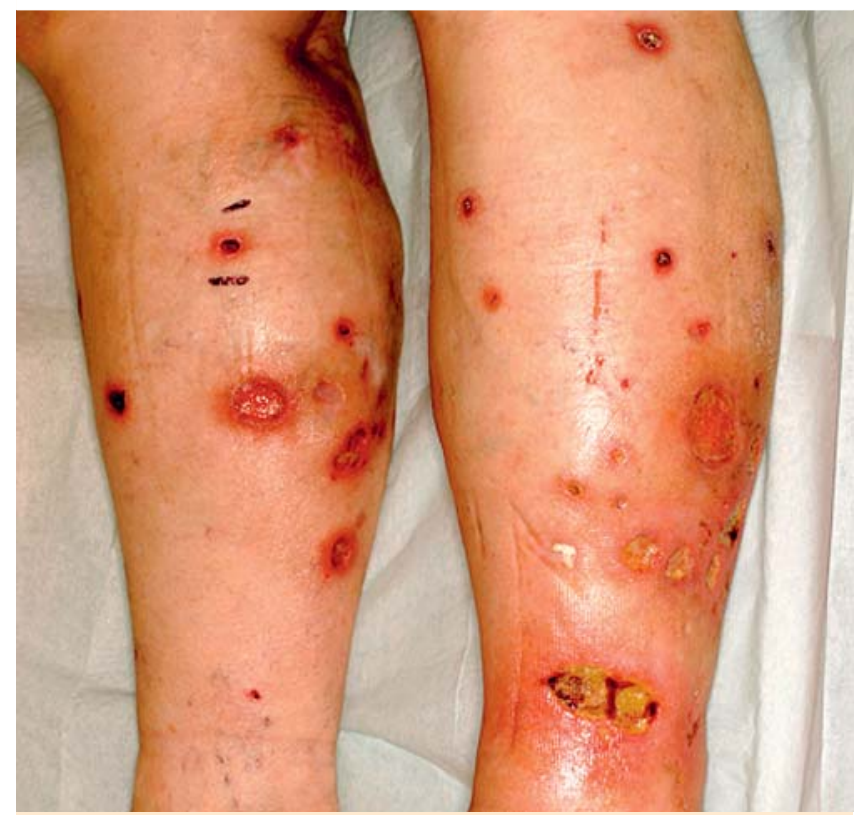

Abb.2 Multiple Ekthymata, die zu therapierefraktären Ulcera crurum geführt haben.

ler Ulzeration die differenzialdiagnostische Abgrenzung beispielsweise zu kutanen Neoplasien oft schwierig, sodass ggf. Biopsien durchgeführt werden sollten. Der klinische Verlauf ist oft chronisch über mehrere Jahre [4]. Weitere seltenere Ursachen eines bakteriell entstandenen Ulcus cruris sind beispielsweise Lues maligna, Diphtherie, Buruli-Ulkus, Mykobakteriose oder Leishmaniose ( Tab.1) [5,6].

\section{Bakterien als Komplikation des chronischen Ulcus cruris \\ $\nabla$}

Wundinfektionen stellen eine gefürchtete Komplikation dar. Es ist jedoch zu beachten, dass bei nahezu allen Wunden eine Vielzahl von Bakterien nachgewiesen werden können, sodass eine unkritische bakterielle Besiedelung oder Kontamination von einer klinisch relevanten Infektion unterschieden werden muss. Man spricht von einer Infektionskrankheit, wenn es nach dem Eindringen von Bakterien in Gewebe zu einer lokalen oder systemischen Wirtsreaktion bis hin zu einer Sepsis kommt. Als Folge von Wundinfektionen stagniert die Wundheilung; oft kommt es sogar zu einer Größenzunahme.

\section{W.A.R.-Score - Wunden mit erhöhtem Infektionsrisiko}

Bei vielen Patienten kann nach klinischer und ggf. serologischer Untersuchung eine Infektionskrankheit diagnostiziert werden. Praktische Schwierigkeiten bereiten Patienten, bei denen noch keine Wundinfektion vorliegt, aber ein erhöhtes Risiko für die Ausbildung einer Wundinfektion besteht. Diese Wunden mit erhöhtem Risiko für Infektionskrankheiten werden auch als „Risikowunden“ bzw. im angloamerikanischen Sprachraum als „wound-at-risk (W.A.R.)“ bezeichnet. Der Einsatz von Antiseptika ist bei Patienten mit unproblematisch kolonisierten Wunden, wenn überhaupt, nur kurzzeitig sinnvoll. Im Gegensatz hierzu ist eine antiseptische Behandlung bei Patienten mit erhöhtem Infektionsrisiko ggf. auch längerfristig erforderlich. Klinisch manifeste Wundinfektionen stellen hingegen nahezu die einzige Indikation für systemische Antibiotikagaben in der Wundbehandlung dar [7]. Eine internationale Expertengruppe hat für die Objektivierung eines erhöhten Infektionsrisikos aktuell mit dem W.A.R.-Score einen Konsensus erarbeitet. Der W.A.R.-Score ist ein Test, bei dem anhand individueller, klinischer Angaben des Patienten ein Punktwert ermittelt wird. Ein Ergebnis von $\geq 3$ Punkten weist auf ein erhöhtes Infektionsrisiko hin. Als klinische Konsequenz wird empfohlen eine antimikrobielle Therapie in das Behandlungskonzept einzubinden. In aktuellen multizentrischen Untersuchungen in Deutschland konnte bei $27 \%$ der Patienten mit chronischem Ulcus cruris ein W.A.R.-Score $\geq 3$ gefunden werden, sodass hier eine antimikrobielle Therapie empfohlen werden kann [8].

\section{Bakteriennachweis \\ $\nabla$}

Es gibt in Deutschland keine akzeptierten Empfehlungen, wann, wie, wo oder wie oft bakteriologische Abstriche bei Patienten mit chronischem Ulcus cruris entnommen werden sollen. In den meisten Wundzentren werden bakteriologische Abstriche zumindest von den Wunden bei Erstvorstellung sowie bei klinischen Hinweisen auf das Vorliegen einer Infektionskrankheit durchgeführt.

Entsprechend der Konsensusvereinbarung der Weltorganisation der Wundheilungsgesellschaften (WUWHS) wurde für bakteriologische Wundabstriche die sogenannte Levine-Technik empfohlen. Hier wird der Abstrich aus einem zirka $1 \mathrm{~cm}^{2}$ großen Areal aus dem Zentrum oder einem klinisch infiziert erscheinenden Areal der Wunde unter leichtem Druck entnommen [9]. Da insbesondere für größere Wunden bekannt ist, dass in verschiedenen Wundbereichen unterschiedliche Bakterienspezies vorkommen, wurde die Levine-Technik in unserer Wundambulanz modifiziert, weiterentwickelt, als „Essener Kreisel“ etabliert und 


\begin{tabular}{|c|c|c|c|c|}
\hline Erkrankung & Erreger & Initiale Chrakteristika & Verlauf & Prädilektionsstellen \\
\hline Ekthymata & $\begin{array}{l}\text { Streptokokken, } \\
\text { Staphylokokken }\end{array}$ & $\begin{array}{l}\text { Pustel auf erythema- } \\
\text { tösem Grund }\end{array}$ & $\begin{array}{l}\text { multiple, scharf begrenzte, } \\
2-5 \mathrm{~cm} \text { große, wie ausge- } \\
\text { stanzt wirkende, wenig } \\
\text { schmerzhafte Ulzerationen }\end{array}$ & untere Körperhälfte \\
\hline $\begin{array}{l}\text { Vegetierende } \\
\text { Pyodermie }\end{array}$ & $\begin{array}{l}\text { Streptokokken, } \\
\text { Staphylokokken }\end{array}$ & $\begin{array}{l}\text { Pustel auf erythema- } \\
\text { tösem Grund }\end{array}$ & $\begin{array}{l}\text { Ulceration mit papillomatö- } \\
\text { sem/verruziformen, teils } \\
\text { unterminiertem Randsaum }\end{array}$ & Extremitäten \\
\hline Lues maligna & $\begin{array}{l}\text { Treponema } \\
\text { pallidum }\end{array}$ & $\begin{array}{l}\text { Ulzeration von } \\
\text { Syphiliden }\end{array}$ & $\begin{array}{l}\text { mehrere, oft wenig schmerz- } \\
\text { hafte Ulzera mit weichen } \\
\text { Rändern }\end{array}$ & generalisiert \\
\hline Hautdiphterie & $\begin{array}{l}\text { Corynebacterium } \\
\text { diphtheriae/ } \\
\text { ulcerans }\end{array}$ & kleine Pustel & $\begin{array}{l}\text { rasch größenprogredientes } \\
\text { Ulkus mit ödematösem } \\
\text { Randsaum }\end{array}$ & Extremitäten \\
\hline Buruli & $\begin{array}{l}\text { Mykobakterium } \\
\text { ulcerans }\end{array}$ & $\begin{array}{l}\text { indolenter subkutaner } \\
\text { Nodus }\end{array}$ & $\begin{array}{l}\text { wenig schmerzhaftes Ulkus } \\
\text { mit tief unterminierten } \\
\text { Rändern }\end{array}$ & Extremitäten \\
\hline
\end{tabular}

Tab. 1 Bakterien als Ursache chronischer Wunden.

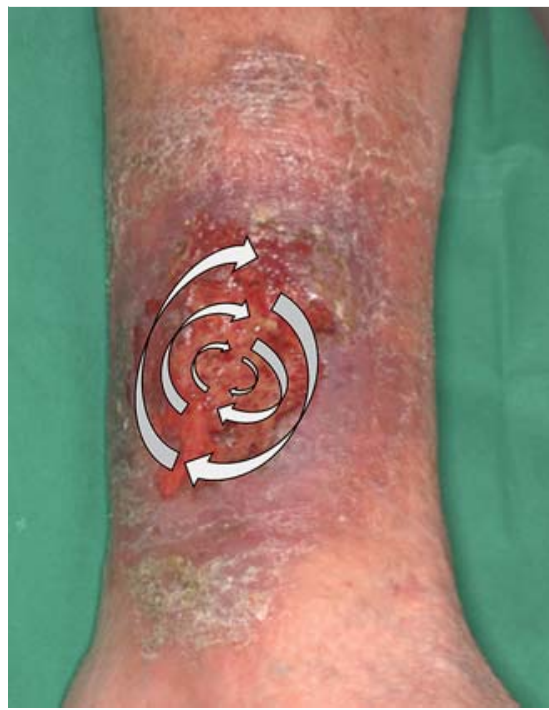

Abb. 3 Bakteriologischer Abstrich von einem oberflächlichen Ulcus cruris venosum entsprechend der Technik „Essener Kreisel“.

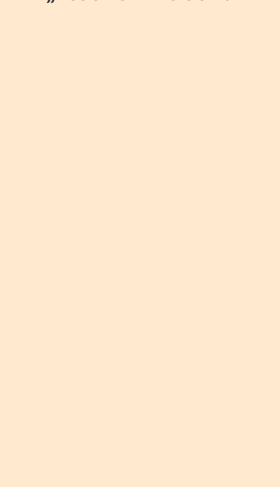

evaluiert. Bei dem „Essener Kreisel“ erfolgt die Entnahme des bakteriologischen Abstriches unter leichtem Druck in Spiralform von außen nach innen, um möglichst alle Areale der Wunde repräsentativ zu erfassen ( $\bullet$ Abb.3). Der Essener Kreisel stellt somit eine leicht anwendbare sowie rasch durchzuführende Modifikation einer konventionellen Abstrichmethode dar, die nur unwesentlich mehr Aufwand bedeutet und in klinischen Untersuchungen zu einem signifikant sensitiveren Nachweis der Keimbesiedlung führte. Der Essener Kreisel eignet sich daher insbesondere für Screeninguntersuchungen beispielsweise zum Ausschluss von multiresistenten Erregern [9]. Eine zusätzliche Biopsieentnahme sollte ausschließlich spezifischen Fragestellungen vorbehalten bleiben $[10,11]$.

\section{Bakterienspektrum des chronischen Ulcus cruris}

In aktuellen eigenen Untersuchungen wurden die Ergebnisse bakteriologischer Abstriche aus zehn dermatologischen Wundambulanzen ausgewertet. Am häufigsten wurde mit 48\% Staphylococcus aureus gefunden; bei $9 \%$ zeigte sich eine Methicillin-Resistenz (MRSA). Pseudomonas aeruginosa wurde bei 31\%, Enterobakterien bei $29 \%$ und Proteus mirabilis bei $14 \%$ der Patienten nachgewiesen. Bei dem regionalen Vergleich fand sich ein signifikantes Süd-Nord-Gefälle für Staphylococcus aureus, Pseudomonas aeruginosa und Enterobakterien. Zudem zeigte sich ein hoch-signifikantes West-Ost Gefälle für MRSA mit Nachweisraten von 14\% im Westen gegenüber $4 \%$ im Osten von Deutschland [12].

\section{Problemkeime im chronischen Ulcus cruris}

Bei den sogenannten „Problemkeimen“ handelt es sich um verschiedene multiresistente Erreger (MRE). Außer MRSA werden hier auch Glycopeptid-resistente Enterokokken (GRE), Vancomycin-resistente Enterokokken (VRE) oder multiresistente gramnegative Erreger (MRGN) zusammengefasst.

\section{Methicillin-resistenter Staphylococcus aureus (MRSA)}

MRSA unterscheidet sich bezüglich Pathogenität und Virulenz meist nicht von Methicillin-sensiblen Staphylococcus aureus (MSSA)-Isolaten. Allerdings ergeben sich durch den MRSA-Nachweis bei Patienten mit Ulcus cruris multiple medizinische und wirtschaftliche Probleme. Grundsätzlich unterscheidet sich die Behandlung der Patienten durch den Nachweis einer klinisch unproblematischen Kolonisation mit MRSA nicht von der anderer chronischer Wunden. Es gilt jedoch aufwendige Hygienemaßnahmen einzuhalten. Bei Nachweis von MRSA ohne klinische Infektionszeichen sollte keine systemische Antibiotikatherapie eingeleitet werden; meist reicht eine topische Therapie beispielsweise mit Antiseptika wie Polihexanid oder Octenidin aus. Die vollständige Eradikation des Keims gestaltet sich bei chronischen Wunden jedoch meist schwierig [13]. Damit MRSA-Keimträger nach Therapieabschluss wieder als MRSA-negativ gelten, müssen mindestens drei negative bakteriologische Abstriche aus zuvor positiven Arealen vorliegen und diese Abstriche in einem Abstand von mindestens 24 Stunden entnommen werden. Die bakteriologischen Kontrollabstriche dürfen frühestens 2-3 Tage nach dem Absetzen der therapeutischen Maßnahmen erfolgen $[14,15]$.

\section{Multiresistente gramnegative Erreger (MRGN)}

Aufgrund der zunehmenden Antibiotikaresistenz-Problematik gramnegativer Erreger hat die Kommission für Krankenhaushygiene und Infektionsprävention (KRINKO) des Robert Koch Instituts (RKI) eine eigene, klinisch orientierte Definition der Multiresistenz gramnegativer Erreger etabliert. Die multiresistenten gramnegativen Bakterien (MRGN) werden nun im Hinblick auf die Anzahl der Resistenzen gegenüber Antibiotikagruppen als 3MRGN beziehungsweise 4MRGN bezeichnet. 
In einer eigenen multizentrischen Untersuchung wurden die bakteriologischen Abstriche von 970 Patienten mit chronischem Ulcus cruris aus dermatologischen Wundambulanzen in Deutschland ausgewertet. Entsprechend der aktuellen KRINKOKlassifikation ließen sich insgesamt acht Patienten mit 4MRGN und 34 Patienten mit 3MRGN identifizieren. Als praktische Konsequenz wird auch in Normalbereichen wie dermatologischen Wundambulanzen oder dermatologischen Stationen zumindest bei Nachweis von 4MRGN die Isolation der Patienten notwendig $[16,17]$.

\section{Pseudomonaden}

Pseudomonas aeruginosa ist das aktuell in Deutschland bei Patienten mit chronischem Ulcus cruris am zweithäufigsten nachgewiesene Bakterium. Aktuelle Studien zeigten, dass die Nachweisraten von Pseudomonas aeruginosa bei Patienten mit chronischem Ulcus cruris innerhalb von 5 Jahren um 10\% auf über 30\% gestiegen sind. Diskutiert wurde, dass die Zunahme dieses „Feuchtkeims“ durch die zunehmende feuchte Wundtherapie und dem wieder weit verbreiteten Einsatz von ungefiltertem Leitungswasser bedingt sein könnte [18].

Die besondere Problematik der Kolonisation mit PseudomonasSpezies besteht darin, dass dies einen eigenständigen wundheilungsbehindernden Faktor darstellt. Es fand sich beispielsweise ein signifikant negativer Einfluss der präoperativen Pseudomonas-Kolonisation der Wunde auf die Einheilraten von Spalthauttransplantaten [19]. Als praktische Konsequenz sollten elektive Eingriffe wie beispielsweise Spalthauttransplantationen erst nach Eradikation der Pseudomonaden durchgeführt werden.

\section{Therapie \\ $\nabla$}

\section{Wundreinigung und Débridement}

Bei den meisten Patienten mit chronischem Ulcus cruris stellen Wundreinigung und/oder Débridement die erste Behandlungsmaßnahme dar [1]. Bei jedem Verbandwechsel ist die Indikation für die Wundreinigung zu prüfen. Die von Expertengremien aktuell empfohlenen Substanzen sind sterile Ringer- oder physiologische Kochsalz-Lösung [2]. Vor allem im ambulanten Sektor wird weiterhin auch Leitungswasser eingesetzt. Vorteile sind die niedrigen Kosten, die ubiquitäre Verfügbarkeit sowie die mechanische Reinigungswirkung bei der Verwendung von Duschköpfen. Der Vorstand der Initiative Chronische Wunde (ICW) e.V. hat aktuell hierzu die verbindliche Rechtslage in Deutschland dargestellt. Die KRINKO hat festgelegt, dass für „offene Wunden Verbandwechsel nur unter aseptischen Vorsichtsmaßnahmen ... durchgeführt werden sollen. Auch jede Spülflüssigkeit muss steril sein ..." Entsprechend dem $§ 23$ Abs. 23 des Infektionsschutzgesetzes sind medizinische Einrichtungen dazu verpflichtet ihre Hygienepläne an diesen aktuellen Hygienestandard anzupassen. Der Einsatz von Leitungswasser ist somit nur dann statthaft, wenn es zuvor mit Filtern mit einer Porengröße von maximal $0,2 \mu \mathrm{m}$ filtriert wird [20].

\section{Débridement}

In der Wundbehandlung bezeichnet der Begriff Débridement die radikale Abtragung von avitalem Gewebe oder Fremdkörpern $[21,1]$. Für das Wunddébridement existieren verschiedene Behandlungsoptionen. Grundsätzlich können chirurgische/physikalische Verfahren von konservativen Methoden unterschieden werden.
Das mechanische beziehungsweise chirurgische Débridement ist für die meisten Patienten mit chronischem Ulcus cruris die Behandlungsoption der ersten Wahl. Zunächst sollte versucht werden mit sterilen Kompressen Beläge abzutragen. Wenn dies nicht ausreichend sein sollte, kann dies beispielsweise mit einem neuen Monofilament-Faserpad (Debrisoft) versucht werden. Mit dem Pad können locker haftender Debris, Exsudat aber auch Bakterien oft schmerzarm aus der Wunde gelöst werden. In einem nächsten Schritt kommen invasive Techniken wie Kürettagen mit dem „scharfen Löffel“ oder einer Ringkürette zum Einsatz. Falls erforderlich, besteht ansonsten auch die Möglichkeit eines chirurgischen Débridements beispielsweise mit einem Dermatom im Sinne eines sogenannten Ulcus-Shave in Voll- oder Teilnarkose [21]. Weitere physikalische Möglichkeiten sind die Anwendung von Ultraschall, Hydrotherapien, bei denen Flüssigkeiten durch Hochdruck auf bis zu $1000 \mathrm{~km} / \mathrm{h}$ beschleunigt werden, oder Lasertherapien [22]. Bei allen physikalischen Maßnahmen ist eine suffiziente Analgesie unerlässlich, die beispielsweise mittels lokalanästhesierender Externa erfolgen kann. Wenn Blutungen nach dem Débridement auftreten, können beispielsweise Alginat- oder Kollagen-haltige Wundauflagen wegen der hämostyptischen Eigenschaften verwendet werden [2].

Als Biochirurgie wird die Behandlung mit steril gezüchteten Fliegenmaden bezeichnet. Neben der schmerzarmen, sehr selektiven Nekrolyse wird hier auch eine effektive Bakterienelimination inklusive MRSA beschrieben [23]. Allerdings soll sich die Biochirurgie weniger gut für die Behandlung von Wunden mit gramnegativen Bakterien eigenen.

Konservative Optionen des Wunddébridements sind beispielsweise Wundverbände mit autolytischen Hydrogelen oder proteolytischen Enzymen. Aktuell kommen Enzympräparate mit Kollagenase oder einer Kombination aus Streptokinase und Streptodornase zum Einsatz [24,2]. Eine weitere, derzeit umstrittene Option ist das osmotische Débridement mit hyperosmolaren Zuckern oder Honig, das für die Patienten mit teils erheblichen Schmerzen verbunden sein kann. Durch den osmotischen Effekt werden allerdings zahlreiche Bakterien effektiv lysiert [25].

\section{Antimikrobielle Therapien}

Die aktuell für den Einsatz bei Patienten mit chronischem Ulcus cruris empfohlenen Antiseptika enthalten meist Polihexanid (Polyhexamethylenbiguanid, PHMB) oder Octenidin. Der Vorteil beider Substanzen ist eine effiziente antimikrobielle Potenz bei geringer Gewebetoxizität. Um eine zuverlässige antimikrobielle Wirksamkeit zu erreichen, ist es wichtig, die empfohlenen Mindesteinwirkzeiten für Octenidin von 2 Minuten und PHMB von 10 Minuten einzuhalten. Hierfür kann die Applikation in Form eines Wundgels oder eingearbeitet in eine Wundauflage sinnvoll sein [2].

Der Einsatz von Wundtherapeutika mit (PVP-)Jod wird aktuell kontrovers diskutiert. So fanden sich in zwei in Deutschland durchgeführten prospektiven Studien bei Patienten mit chronischem Ulcus cruris bei $20 \%$ beziehungsweise bei $16 \%$ der Patienten Kontaktsensibilisierungen auf PVP-Jod [26,27]. Zudem wurden für die aktuell eingesetzten Konzentrationen zytotoxische Eigenschaften sowie Eiweißfehler beschrieben. Daher sollten Jodverbindungen bei Patienten mit chronischen Wunden - wenn überhaupt - nur kurzzeitig bei frustranen Behandlungen gramnegativer Bakterien eingesetzt werden [28].

Eine weitere Alternative ist die seit mehreren Jahren stark zunehmende Verbreitung von Wundauflagen mit Silber. Da hier viele 
unterschiedliche Silberzubereitungen in sehr unterschiedlichen Mengen und Bindungsformen eingesetzt werden, ist die wissenschaftliche Datenlage unzureichend, sodass aktuell keine verbindliche Empfehlung für die praktische Anwendung von Wundauflagen mit Silber gegeben werden kann [29].

\section{Fazit}

\section{$\nabla$}

Bakterien spielen in der Genese und Therapie von Patienten mit chronischem Ulcus cruris eine zentrale Rolle und sollten daher bei der Diagnostik ebenso wie bei der Therapieplanung berücksichtigt werden.

\section{Interessenkonflikt}

$\nabla$

Vorträge, Studien und/oder Beratungen folgender Firmen: 3M, B. Braun, BSN, Coloplast, Convatec, Draco, Hartmann, KCI, Lohmann \& Rauscher, Medoderm, Sastomed, Serag Wiessner, Systagenix, UCB-Pharma, Urgo

\section{Abstract}

\section{Clinical Relevance of Bacteria in Chronic Leg Ulcers $\nabla$}

Bacteria in chronic leg ulcers may be central relevant in both the genesis and as a complication. Staphylococcus aureus is with a current detection rate of $50 \%$ the germ detected most often. The second highest detection rate with increasing tendency is found for Pseudomonas aeruginosa. Problematic germs like Methicillinresistant Stapylococcus aureus (MRSA) or multiresistant gram negative bacteria (MRGN) are gaining increasingly more importance. A bacteriological swab taken from the wound-surface in an Essen Rotary technique is in most cases adequate for diagnostics.

Most of the patients with chronic leg ulcers have a clinically unproblematic bacterial colonization, so that the administration of systemic antibiotics usually is not required. Patients with increased risk of infection, critical colonization, or local signs of infection should be treated with antiseptics containing polihexanid or octenidine.

\section{Literatur}

1 AWMF, S3-Leitlinie der Deutschen Gesellschaft für Wundheilung und Wundbehandlung: Lokaltherapie chronischer Wunden bei Patienten mit den Risiken periphere arterielle Verschlusskrankheit, Diabetes mellitus, chronisch venöse Insuffizienz. http://www.awmf.org/uploads/tx_szleitlinien/091-0011_S3_Lokaltherapie_chronischer_Wunden_2012-06.pdf

2 Dissemond J. Ulcus cruris - Genese, Diagnostik und Therapie. 4. Aufl. Bremen: UNI-MED Verlag; 2012

3 Körber A, Klode J, Al-Benna $S$ et al. Etiology of chronic leg ulcers in 31,619 patients in Germany analyzed by an expert survey. J Dtsch Dermatol Ges 2011; 9: 116-121

4 Dissemond J. Chronisch vegetierende Pyodermie. ZfW 2004; 9: 294295

5 Körber A, Dissemond J, Hillen U et al. HIV-positive patient with multiple ulcers. Lues maligna. Hautarzt 2003; 54: $1098-1102$

6 Zeegelaar JE, Faber WR. Imported tropical infectious ulcers in travelers. Am J Clin Dermatol 2008; 9: 219-232
7 O'Meara S, Al-Kurdi D, Ologun Y et al. Antibiotics and antiseptics for venous leg ulcers. Cochrane Database Syst Rev 2010, CD003557

8 Jockenhöfer F, Gollnick H, Herberger $K$ et al. W.A.R. scores in patients with chronic leg ulcers: results of a multicentre study. J Wound Care 2014; 23: 5-12

9 Al Ghazal P, Körber A, Klode J et al. Evaluation of the Essen Rotary as a new technique for bacterial swabs: results of a prospective controlled clinical investigation in 50 patients with chronic leg ulcers. Int Wound J 2014; 11: $44-49$

10 Davies CE, Hill KE, Newcombe RG et al. A prospective study of the microbiology of chronic venous leg ulcers to reevaluate the clinical predictive value of tissue biopsies and swabs. Wound Repair Regen 2007; 15: $17-22$

11 Gjodsbol K, Skindersoe ME, Christensen JJ et al. No need for biopsies: comparison of three sample techniques for wound microbiota determination. Int Wound J 2012; 9: 295-302

12 Jockenhöfer F, Gollnick H, Herberger $K$ et al. Bacteriological pathogen spectrum of chronic leg ulcers: Results of a multicenter trial in dermatologic wound care centers differentiated by regions. J Dtsch Dermatol Ges 2013; 11: 1057-1063

13 Rietkötter J, Körber A, Grabbe S et al. Eradication of methicillin-resistant Staphylococcus aureus in a chronic wound by a new polyhexanide hydrogel. J Eur Acad Dermatol Venereol 2007; 21: 1416-1417

14 Dissemond J. Methicillin resistant Staphylococcus aureus (MRSA): Diagnostic, clinical relevance and therapy. J Dtsch Dermatol Ges 2009; 7: $544-551$

15 Mattner F, Bange FC, Meyer E et al. Preventing the spread of multidrugresistant gram-negative pathogens: recommendations of an expert panel of the German Society For Hygiene and Microbiology. Dtsch Arztebl Int 2012; 109: 39-45

16 Jockenhöfer F, Gollnick H, Herberger $K$ et al. Aktuelle Nachweisraten multiresistenter gramnegativer Bakterien (3MRGN, 4MRGN) bei Patienten mit chronischem Ulcus cruris. Hautarzt [in press]

17 Commission recommendation for hospital hygiene and infection prevention (KRINKO) at the Robert Koch Institute (RKI). Hygiene measures for infection or colonization with multidrug-resistant gram-negative bacilli. Bundesgesundheitsblatt Gesundheitsforschung Gesundheitsschutz 2012; 55: $1311-1354$

18 Körber A, Schmid EN, Buer J et al. Bacterial colonization of chronic leg ulcers: current results compared with data 5 years ago in a specialized dermatology department. J Eur Acad Dermatol Venereol 2010; 24: $1017-1025$

19 Hogsberg T, Bjarnsholt T, Thomsen JS et al. Success rate of split-thickness skin grafting of chronic venous leg ulcers depends on the presence of Pseudomonas aeruginosa: a retrospective study. PLoS One 2011; 6: e20492

20 Schwarzkopf A, Assenheimer B, Bültemann A et al. für den Vorstand der Initiative Chronische Wunde e.V. Hygienefachliche und -rechtliche Bewertung der Anwendung von Leitungswasser als Wundspüllösung. Wundmanangement 2012; 6: 195-197

21 Strohal R, Dissemond J, Jordan O'Brien J et al. The EWMA document: Debridement. J Wound Care 2013; 22: 5

22 Klötgen K, Klode J, Körber A et al. Ultraschall in der Therapie chronischer Wunden. Akt Dermatol 2009; 35: 237-242

23 Dissemond J, Koppermann M, Esser $S$ et al. Treatment of methicillin-resistant Staphylococcus aureus (MRSA) as part of biosurgical management of a chronic leg ulcer. Hautarzt 2002; 53: 608-612

24 Dissemond J, Goos M. Konditionierung chronischer Wunden. Hautarzt 2003; 54: 1073 - 1079

25 Jull $A B$, Rodgers A, Walker N. Honey as a topical treatment for wounds. Cochrane Database Syst Rev 2008: CD005083

26 Lehnen M, Kohaus S, Körber A et al. Contact allergies in patients with chronic wounds: results of a study from 1999 to 2004. Hautarzt 2006; 57: $303-306,308$

27 Reich-Schupke S, Kurscheidt J, Appelhans $C$ et al. Patch testing in patients with leg ulcers with special regard to modern wound products. Hautarzt 2010; 61: 593-597

28 Kramer A, Daeschlein G, Kammerlander $G$ et al. Konsensusempfehlung zur Auswahl von Wirkstoffen für die Wundantiseptik. ZfW 2004; 3: $110-120$

29 Storm-Versloot MN, Vos CG, Ubbink DT et al. Topical silver for preventing wound infection. Cochrane Database Syst Rev 2010, CD006478 